EUROPEAN JOURNAL OF PURE AND APPLIED MATHEMATICS

Vol. 14, No. 4, 2021, 1237-1248

ISSN 1307-5543 - ejpam.com

Published by New York Business Global

\title{
Chatterjee and Extension of Chatterjee Fixed Point Theorems on Operators on Hilbert C*-Modules
}

\author{
Rashwan. A. Rashwan ${ }^{1, *}$, Howida Adel AlFran², Asmaa Fangary ${ }^{3}$, Saleh \\ Omran $^{3}$ \\ 1 Department of Mathematics, Faculty of Science, Assuit University, Assuit, Egypt \\ 2 Department of Mathematics, Al-Leith University College, Umm Al Qura University, \\ Kingdom of Saudi Arabia \\ 3 Department of Mathematics, Faculty of Science, South Valley University, Qena, Egypt
}

\begin{abstract}
In this paper we consider some fixed point theorem (such as Chatterjee and extension of Chatterjee) in operators of Hilbert $C^{*}$-modules, based on a definition of valued operator Hilbert $\mathrm{C}^{*}$-modules normed space. Also We give some examples to clear our definitions.
\end{abstract}

2020 Mathematics Subject Classifications: 47H10, 46L05, 46L08

Key Words and Phrases: Fixed Point Theorems, $C^{*}$-algebra, Operators on Hilbert $C^{*}$-modules

\section{Introduction}

Hilbert $C^{*}$-modules consider a mathematical objects where generalize the notion of a Hilbert space by allowing the inner product to take values in a (commutative, unital) $C^{*}$-algebra rather than in the field of complex numbers. Hilbert $C^{*}$-modules were first introduced in 1953 by Kaplansky [5]. Later, the theory was developed independently by Paschke [12] and Rieffel [16] where the research on Hilbert $C^{*}$-modules began in the 70' $s$ in the work of the induced representations of $C^{*}$-algebras by M. A. Rieffel [16] also Kasparov [6] introduced the definition of $K K$-theory by using Hilbert $C^{*}$-modules

$\mathrm{C}^{*}$-algebra is a main subject in the functional analysis and the operator theory which play fundamental role in noncommutative geometry and theoretical physics, especially the quantum mechanics

Ma and et al. [20], introduced the concept of $C^{*}$-algebra-valued metric spaces. The main idea consists in using the set of all positive elements of a unital $C^{*}$-algebra instead

${ }^{*}$ Corresponding author.

DOI: https://doi.org/10.29020/nybg.ejpam.v14i4.4071

Email addresses: rashwan10@gmail.com (R. A. Rashwan), hafran@uqu.edu.sa (H. Adel AlFran), Asmaa.fangary44@yahoo.com (A. Fangary),salehomran@yahoo.com (S. Omran) 
of the set of real numbers. They presented some fixed point results for mapping under contractive or expansive conditions in these spaces. Later, Ma and et al. [21], introduced the concept of $C^{*}$-algebra-valued b-metric spaces and proved some fixed point theorems such as Banach and Kannan type fixed point theorems.For other results on $C^{*}$-algebravalued b-metric spaces and $C^{*}$-algebra-valued-metric spaces, see $[4,13,15,18,22]$.

An element $x \in \mathbb{A}$ is a positive element, denote it by $x \succeq 0$, if $x \in \mathbb{A}_{h}$ and $\sigma(x) \subset[0,+\infty]$, where $\sigma(x)$ is the spectrum of $x$ and $\mathbb{A}_{h}=\left\{x \in \mathbb{A}: x^{*}=x\right\}$. Using positive elements, one can define a partial ordering $\preceq$ on $\mathbb{A}_{h}$ as follows: $x \preceq y$ if and only if $y-x \succeq 0$. From now on, by $\mathbb{A}_{+}$we denote the set $\{x \in \mathbb{A}: x \succeq 0\}$ and $|x|=\left(x^{*} x\right)^{\frac{1}{2}}$.

\section{Preliminaries}

In this section, we begin with some basic notations and definition $C^{*}$-algebra and fixed point theory that will be very important and useful in the sequal.

Definition 1. [9] A Banach $*$-algebra is a *-algebra $\mathbb{A}$ together with a complete submultiplicative norm such that $\|a b\| \leq\|a\|\|b\| \quad$ (for all $a, b \in \mathbb{A}$ ). A $C^{*}$ algebra is a Banach *-algebra such that $\left\|a^{*} a\right\|=\|a\|^{2} \quad$ (for all $\left.a \in \mathbb{A}\right)$.

Definition 2. [9] An element $a \in \mathbb{A}$ is positive element, if $a=a^{*}$ and $\sigma(a) \subseteq \mathbb{R}^{+}$, where $\sigma(a)$ is the spectrum of $a$, we denote $\mathbb{A}_{+}$the set of all positive element in $\mathbb{A}$.

Definition 3. $[\mathbf{8}, \mathbf{1 9}]$ A pre-Hilbert $C^{*}$-module $\mathcal{E}$ over a $C^{*}$-algebra $\mathbb{A}$, is a right $\mathbb{A}$-module together with an $\mathbb{A}$-valued inner product $\langle., .>: \mathcal{E} \times \mathcal{E} \longrightarrow \mathbb{A}$ satisfying the conditions:

(1) $<x, x>\succeq 0$ for all $x \in \mathcal{E}$;

(2) $\langle x, x>=0$ if and only if $x=0$;

(3) $<x, \alpha y+\beta z>=\alpha<x, y>+\beta<x, z>$ for all $x, y, z \in \mathcal{E}, \alpha, \beta \in \mathbb{C}$;

(4) $\langle x, y a>=<x, y>a$ for all $x, y \in \mathcal{E}, a \in \mathbb{A}$;

(5) $<x, y>^{*}=<y, x>$ for all $x, y \in \mathcal{E}$.

Definition 4. [8] The norm of an element $e \in \mathcal{E}$ is defined as

$$
\|x\|_{\mathcal{E}}:=\sqrt{\|<x, x\rangle \|_{\mathbb{R}}} \text {, where }\|\cdot\|_{\mathbb{R}} \text { is the } \mathbb{R} \text {-valued norm. }
$$

If a pre-Hilbert $\mathbb{A}$-module is complete with respect to its norm, it is said to be a Hilbert $\mathbb{A}$ -module.

Example 1. Every $C^{*}$-algebra $\mathbb{A}$ is a Hilbert $\mathbb{A}$-module over itself when equipped with the $\mathbb{A}$-valued inner product given simply by

$$
<a, b>=a^{*} b,(a, b \in \mathbb{A}) .
$$

Definition 5. [19] Let $\mathcal{E}$ be a Hilbert $\mathbb{A}$-module. A map $T: \mathcal{E} \longrightarrow \mathcal{E}$ is said to be adjointable if there exists a map $T^{*}: \mathcal{E} \longrightarrow \mathcal{E}$ satisfying 


$$
<x, T y>=<T^{*} x, y>
$$

for all $x, y \in \mathcal{E}$.

Definition 6. [3] An element $T \in l(\mathcal{E})$ is positive if for every $x \in \mathcal{E}$ we have $\left\langle T x, x>_{\mathbb{A}} \succeq\right.$ 0 and we write it by $T \succeq 0$ and we denote the $\operatorname{set} l(\mathcal{E})_{+}=\{T \in \mathcal{E} ; T \succeq 0\}$, we define a partial ordering relation on $l(\mathcal{E})_{+}$as

$$
\text { if } T_{1}, T_{2} \in l(\mathcal{E}), T_{1} \preceq_{l(\mathcal{E})} T_{2} \text { if and only if } T_{2}-T_{1} \in l(\mathcal{E})_{+}
$$

Definition 7. [3] $l(\mathcal{E})=\{T: \mathcal{E} \longrightarrow \mathcal{E}\}$ is the set of all adjiontable linear operators with $\|T\|=\sup \left\{\|T x\|_{\mathcal{E}} ;\|x\|_{\mathcal{E}} \leq 1\right\}$ is a $C^{*}$-algebra.

\section{Main Results}

Definition 8. Let $l(\mathcal{E})_{+}$be a subset of $l(\mathcal{E}) . l(\mathcal{E})_{+}$is called Cone of $l(\mathcal{E})$ if and only if :

(1) $l(\mathcal{E})_{+} \cap\left(-l(\mathcal{E})_{+}\right)=\left\{0_{l(\mathcal{E})}\right\},\left(0_{l(\mathcal{E})}\right.$ is the zero vector $)$;

(2) $l(\mathcal{E})_{+}$is closed in $l(\mathcal{E})$;

(3) $T a+S b \in l(\mathcal{E})_{+} ; a T+b S \in l(\mathcal{E})_{+} a, b \in A, T \lambda+S \beta \in l(\mathcal{E})_{+}: \lambda, \beta \in \mathbb{C}$;

(4) $l(\mathcal{E})_{+} \cdot l(\mathcal{E})_{+} \subseteq l(\mathcal{E})_{+}$.

Definition 9. An $l(\mathcal{E})$-valued metric on a set $X$ is a function $d_{l(\mathcal{E})}: X \times X \longrightarrow l(\mathcal{E})$ such that for all $x, y$ and $z$ in $X$ the following conditions are hold:

(1) $d_{l(\mathcal{E})}(x, y) \succeq 0$;

(2) $d_{l(\mathcal{E})}(x, y)=0$ if and only if $x=y$;

(3) $d_{l(\mathcal{E})}(x, y)=d_{l(\mathcal{E})}(y, x)$;

(4) $d_{l(\mathcal{E})}(x, y) \preceq d_{l(\mathcal{E})}(x, z)+d_{l(\mathcal{E})}(z, y)$.

Then the triple $\left(X, l(\mathcal{E}), d_{l(\mathcal{E})}\right)$ is called an $l(\mathcal{E})$-valued metric space.

Definition 10. [20] Let $X$ be a nonempty set. Suppose the mapping $d: X \times X \longrightarrow \mathbb{A}$ satisfies:

(1) $0_{\mathbb{A}} \preceq d(x, y)$ for all $x, y \in X$ and $d(x, y)=0_{\mathbb{A}}$ if and only if $x=y$.

(2) $d(x, y)=d(y, x)$ for all $x, y \in X$.

(3) $d(x, y) \preceq d(x, z)+d(z, y)$ for all $x, y, z \in X$.

Then $d$ is called a $C^{*}$-algebra-valued metric on $X$ and $(X, \mathbb{A}, d)$ is a $C^{*}$-algebra-valued metric space. 
Definition 11. Let $\left(X, l(\mathcal{E}), d_{l(\mathcal{E})}\right)$ be an $l(\mathcal{E})$-valued metric spacs. Suppose that $x_{n} \subset X$ and $x \in X$ If for any $\varepsilon_{l(\mathcal{E})} \succ 0_{l(\mathcal{E})}$ (where $0_{l(\mathcal{E})}$ is the zero element in $l(\mathcal{E})$ ) there exists $N \in \mathbb{N}$ such that for all $n>N, d_{l(\mathcal{E})}\left(x_{n}, x\right) \preceq \varepsilon_{l(\mathcal{E})}$, then $\left\{x_{n}\right\}$ is said to be converge with respect to $l(\mathcal{E})$, and $\left\{x_{n}\right\}$ converges to $x$ and $x$ is the limit of $\left\{x_{n}\right\}$. We denote it by $\lim _{n \longrightarrow+\infty}\left\{x_{n}\right\}=x$.

If for any $\varepsilon_{l(\mathcal{E})} \succ 0_{l(\mathcal{E})}$ there exists $N \in \mathbb{N}$ such that for all $n, m>N, d\left(x_{n}, x_{m}\right) \preceq \varepsilon_{l(\mathcal{E})}$, then $\left\{x_{n}\right\}$ is said to be a Cauchy with respect to $l(\mathcal{E})$.

We say $\left(X, l(\mathcal{E}), d_{l(\mathcal{E})}\right)$ is a complete $l(\mathcal{E})$ - valued metric spacs if every Cauchy sequence with respect to $l(\mathcal{E})$ is convergent.

Lemma 1. A sequence $x_{n} \subset X$ is convergence if $\left\|x_{n}\right\| \longrightarrow 0$ forall $n>N$ such that $N \in \mathbb{N}$.

Example 2. Let $X=\mathbb{A}^{\oplus n}, \mathcal{E}=\mathbb{A}^{\oplus n}$ and $l(\mathcal{E})=\left\{T: \mathbb{A}^{\oplus n} \longrightarrow \mathbb{A}^{\oplus n}: T\left(a_{1}, a_{2}, \ldots, a_{n}\right)=\right.$ $\left.\left(T a_{1}, T a_{2}, \ldots, T a_{n}\right)\right\}$. Define

$$
d\left(\left(a_{1}, a_{2}, \ldots, a_{n}\right),\left(b_{1}, b_{2}, \ldots, b_{n}\right)\right)=\left(\left\|T a_{1}-T b_{1}\right\|_{\mathbb{R}},\left\|T a_{2}-T b_{2}\right\|_{\mathbb{R}}, \ldots,\left\|T a_{n}-T b_{n}\right\|_{\mathbb{R}}\right) I_{\mathbb{A}},
$$

where $\left(a_{1}, a_{2}, \ldots, a_{n}\right),\left(b_{1}, b_{2}, \ldots, b_{n}\right) \in \mathbb{A}^{\oplus n}$ and $I_{\mathbb{A}}$ is the identity element of $\mathbb{A}$. It is easy to verify that $d_{l(\mathcal{E})}$ is an $l(\mathcal{E})$-valued metric space and $\left(X, \mathbb{A}^{\oplus n}, d_{l(\mathcal{E})}\right)$ is a complete $l(\mathcal{E})$ -valued metric space, since $\mathbb{A}$ is complete.

Definition 12. let $(X, l(\mathcal{E}))$ is an $l(\mathcal{E})$-metric space, we define the open ball on $X$

$$
B_{l(\mathcal{E})}\left(a, \epsilon_{l(\mathcal{E})}\right)=\left\{x \in X ;\|x-a\| \prec \epsilon_{l(\mathcal{E})}\right\}
$$

Definition 13. Suppose that $\left(X, d_{l(\mathcal{E})}\right)$ is $l(\mathcal{E})$-metric space, let $x \in X$ then a neighhborhood of $x$ is any set containing $B_{l(\mathcal{E})}\left(x, \epsilon_{l(\mathcal{E})}\right)$ for some $\epsilon_{l(\mathcal{E})} \succ 0_{l(\mathcal{E})}$.

Definition 14. Suppose that $\left(X, d_{l(\mathcal{E})}\right)$ is $l(\mathcal{E})$-metric space, a subset $U \subset X$ is open if for every $x \in U$ there exist an open ball $B_{l(\mathcal{E})}\left(a, \epsilon_{l(\mathcal{E})}\right)$ such that $x \in B_{l(\mathcal{E})}\left(x, \epsilon_{l(\mathcal{E})}\right) \subset U$.

Motivaied by the idea in [7],[17],[9], we give the following definations.

Definition 15. Let $X$ be vector space, if the function $\|\cdot\|_{l(\mathcal{E})}: X \longrightarrow l(\mathcal{E})$ has the following properties:

(1) $\|x\|_{l(\mathcal{E})} \succeq 0$ i.e $\|x\|_{l(\mathcal{E})}$ is a positive operator, $\|x\|_{l(\mathcal{E})}=0$ if and only if $x=0$;

(2) $\|\lambda x\|_{l(\mathcal{E})}=|\lambda|\|x\|_{l(\mathcal{E})} ; \lambda \in \mathbb{C}$;

(3) $\|x+y\|_{l(\mathcal{E})} \preceq\|x\|_{l(\mathcal{E})}+\|y\|_{l(\mathcal{E})}$.

Then $\|$.$\| is said to be l(\mathcal{E})$-valued norm defined on $X$, and $(X,\|\|$.$) is said to be l(\mathcal{E})$-valued normed $l(\mathcal{E})$ space.

Also we will set the relation between $l(\mathcal{E})$-valued metric space and $l(\mathcal{E})$-valued normed space as follow $\quad d_{l(\mathcal{E})}(x, y)=\|x-y\|_{l(\mathcal{E})}$. 
Definition 16. Let $X$ be a vector space over a field $(F=\mathbb{C}, \mathbb{R})$ we say that $X$ is a right $l(\mathcal{E})$-vector space if satisfy:

(1) $(x+y) T=x T+y T$;

(3) $x\left(T_{1}+T_{2}\right)=x T_{1}+x T_{2}$;

(3) $(x S) T=x(S T)$.

Where $x, y \in X$ and $S, T \in l(\mathcal{E})$.

Lemma 3.2 Let $X$ be a right $l(\mathcal{E})$-vector space then,

$$
\|x T\|_{l(\mathcal{E})} \preceq\|x\|\|T\|_{l(\mathcal{E})} .
$$

Proof. $\|x T\|^{2}=\sup _{\|x\|=1}\{<x T, x T>, x \in \mathcal{E}\} \leq\|x\|\|T\|_{l(\mathcal{E})}$.

Definition 17. Let $\mathbb{A}$ be $C^{*}$-algebra,and $l(\mathcal{E})$ be an $l(\mathcal{E})$-normed spac. We say that $l(\mathcal{E})$ is right $\mathbb{A}$-module if the mapping is right module multiplication $(a, T) \longmapsto x a$ of $\mathbb{A} \times l(\mathcal{E}) \longrightarrow$ $l(\mathcal{E})$ such that the following axioms are satisfied:

(1) For each fixed $a \in \mathbb{A}$ the map $(a, T) \longrightarrow$ Ta is linear on $l(\mathcal{E}): T \in l(\mathcal{E})$;

(2) For each fixed $T \in l(\mathcal{E})$ the map $(a, T) \longrightarrow T a$ is linear on $\mathbb{A}$;

(3) For all $a_{1}, a_{2} \in \mathbb{A}$ and all $T \in l(\mathcal{E})$ we have that $\left(T a_{1}\right) a_{2}=T\left(a_{1} a_{2}\right)$.

Example 3. If we define the norm $\|x\|_{l(\mathcal{E})}=\|x\| I_{l(\mathcal{E})}$ (where $I_{l(\mathcal{E})}$ is the identity operator of $l(\mathcal{E})$ ) then we have that $l(\mathcal{E})$ with this norm is $l(\mathcal{E})$-norm.

Lemma 2. If $T$ is positive if and only if $T^{*}$ is positive.

Proof. Let $*: \mathbb{A} \longrightarrow \mathbb{A}$ is $*$-homomorphism.

if $T^{*}$ is positive implies $\left\langle T^{*} x, x\right\rangle \succeq 0$ implies $\langle x, T x\rangle \succeq 0$ implies $\langle x, T x\rangle^{*} \succeq 0$ implies $\langle T x, x\rangle \succeq 0$ implies $T$ is positive.

$\Longleftarrow$ if $\mathrm{T}$ is positive implies $\langle T x, x\rangle \succeq 0$ implies $\left\langle x, T^{*} x\right\rangle \succeq 0$ implies $\left\langle x, T^{*} x\right\rangle^{*} \succeq 0$ implies $<T^{*} x, x>\succeq 0$ implies $T^{*} \succeq 0$ implies $T^{*}$ is positive.

Lemma 3. If $S$ is positive operator then for any operator $T$ implies $T^{*} S T$ is positive operator.

Proof. Since $S \succeq 0$, we can write $S=R^{*} R$, for any $R \in\left(l_{\mathcal{E}}\right)$ implies $T^{*}\left(R^{*} R\right) T=$ $\left(T^{*} R^{*}\right)(R T)=(R T)^{*}(R T) \succeq 0$

Definition 18. A sequence $\left\{x_{n}\right\}$ in $X$ is said to be convergent if for every $\epsilon>0$, there is a natural number $N$ such that for $n>N$ we have 


$$
\left.\left\|x_{n}-x\right\| \preceq_{l(\mathcal{E})} \epsilon I_{l(\mathcal{E})} \text { (where } I_{l(\mathcal{E})} \text { the identity operator of } l(\mathcal{E})\right) \text {. }
$$

Definition 19. A sequence $\left\{x_{n}\right\}$ in $X$ is said to be a Cuachy sequence if for every $\epsilon>0$, there is a natural number $N$ such that for $n, m>N$ we have

$$
\left\|x_{n}-x_{m}\right\| \preceq_{l(\mathcal{E})} \epsilon I_{l(\mathcal{E})} .
$$

Lemma 4. A sequence $\left\{x_{n}\right\}$ in $X$ is convergence in $X$ if $\left\|x_{n}\right\|_{\mathbb{R}} \longrightarrow 0$ at $n \longrightarrow+\infty$.

Proof. Since in $\left.l_{(} \mathcal{E}\right)$ - valued metric spacs. We say that a sequance $x_{n} \subset X$ converges to $x \in X$ If for any $\varepsilon_{l(\mathcal{E})} \succ 0_{l(\mathcal{E})}$ (where $0_{l(\mathcal{E})}$ is the zero element in $l(\mathcal{E})$ ) there exists $N \in \mathbb{N}$ such that for all $n>N, d_{l(\mathcal{E})}\left(x_{n}, x\right) \preceq \varepsilon_{l(\mathcal{E})}$, then this implies $\left\|d_{l(\mathcal{E})}\left(x_{n}, x\right)\right\|_{\mathbb{R}}<\mathcal{E}, \quad \mathcal{E} \in \mathbb{R}$.

Lemma 5. [2, 9] Suppose that $\mathbb{A}$ is a unital $C^{*}$-algebra with a unit $I$ :

(1) for any $x \in \mathbb{A}_{+}$we have $x \preceq I$ if and only if $\|x\| \leq 1$;

(2) If $a \in \mathbb{A}_{+}$with $\|a\|<\frac{1}{2}$, then $I-a$ is invertable and $\left\|a(I-a)^{-1}\right\|<1$;

(3) suppose that $a, b \in \mathbb{A}$ with $a, b \succeq 0$ and $a b=b a$, then $a b \succeq 0$.

(4) by $\mathbb{A}$ we denote the set $\{a \in \mathbb{A}: a b=b a$ forall $b \in \mathbb{A}\}$ Let $a \in \mathscr{A}$, if $b, c \in \mathbb{A}$ with $b \succeq c \succeq 0$

$$
(I-a)^{-1} b \succeq(I-a)^{-1} c .
$$

Definition 20. Let $\left(X, l(\mathcal{E}),\|\cdot\|_{l(\mathcal{E})}\right)$ be an $l(\mathcal{E})$ normed space. We call a mapping $T$ : $X \longrightarrow X$ is $l(\mathcal{E})$ contractive mapping on $X$ if there exists an $M \in l(\mathcal{E})$ with $\|M\|_{l(\mathcal{E})} \leq 1$ such that

$$
\|T x-T y\|_{l(\mathcal{E})} \preceq M^{*}\|x-y\|_{l(\mathcal{E})} M \text { forall } \quad x, y \in X .
$$

Definition 21. An $l(\mathcal{E})$ - Banach space is a complete $l(\mathcal{E})$-normed space $\left(X,\|\cdot\|_{l(\mathcal{E})}\right)$.

Many results on fixed point theorems have been extended from metric spaces to $C^{*}$-algebra valued metric spaces with different contraction conditions (see for example $[20],[9],[10],[11],[14])$

Theorem 1. (Chatterjee Type theorem $[\mathbf{1}])$ Let $\left(X, l(\mathcal{E}),\|\cdot\|_{l(\mathcal{E})}\right)$ be an $l(\mathcal{E})$ complete normed space and $T: X \longrightarrow X$ be a self mapping satisfy the following contraction condition

$$
\|T x-T y\|_{l(\mathcal{E})} \preceq \frac{M}{2}\left[\quad\|T x-y\|_{l(\mathcal{E})}+\|T y-x\|_{l(\mathcal{E})}\right],
$$

where $M \in(l(\mathcal{E}))_{+}$with $\|M\|_{l(\mathcal{E})}<1$, Then $T$ has a unique fixed point.

Proof. Let $x_{0} \in X$ be arbitrary point and construct a sequence $\left\{x_{n}\right\}_{n=0}^{+\infty} \subseteq X$ by the way: $x_{1}=T x_{0}, x_{2}=T x_{1}, \ldots ., x_{n+1}=T x_{n}$ 
R. A. Rashwan et al. / Eur. J. Pure Appl. Math, 14 (4) (2021), 1237-1248

$$
\begin{aligned}
\left\|x_{n+1}-x_{n}\right\|_{l(\mathcal{E})} & =\left\|T x_{n}-T x_{n-1}\right\|_{l(\mathcal{E})} \\
& \preceq \frac{M}{2}\left[\quad\left\|T x_{n}-x_{n-1}\right\|_{l(\mathcal{E})}+\left\|T x_{n-1}-x_{n}\right\|_{l(\mathcal{E})} \quad\right] \\
& =\frac{M}{2}\left[\quad\left\|x_{n+1}-x_{n-1}\right\|_{l(\mathcal{E})}+\left\|x_{n}-x_{n}\right\|_{l(\mathcal{E})} \quad\right] \\
& \preceq \frac{M}{2}\left[\quad\left\|x_{n+1}-x_{n}\right\|_{l(\mathcal{E})}+\left\|x_{n}-x_{n-1}\right\|_{l(\mathcal{E})} \quad\right] \\
& \preceq \frac{M}{2}\left\|x_{n+1}-x_{n}\right\|_{l(\mathcal{E})}+\frac{M}{2}\left\|x_{n}-x_{n-1}\right\|_{l(\mathcal{E})} .
\end{aligned}
$$

Thus,

$\left(I_{l(\mathcal{E})}-\frac{M}{2}\right)\left\|x_{n+1}-x_{n}\right\|_{l(\mathcal{E})} \preceq \frac{M}{2}\left\|x_{n}-x_{n-1}\right\|_{l(\mathcal{E})}$.

Since $M \in(l(\mathcal{E}))_{+}$with $\left\|\frac{M}{2}\right\|_{l(\mathcal{E})} \preceq \frac{1}{2}$, one have $\left(I_{l(\mathcal{E})}-\frac{M}{2}\right)^{-1} \in(l(\mathcal{E}))_{+}$, and furthermore $\frac{M}{2}\left(I_{l(\mathcal{E})}-\frac{M}{2}\right)^{-1} \in(l(\mathcal{E}))_{+}$with $\left\|\frac{M}{2}\left(I_{l(\mathcal{E})}-\frac{M}{2}\right)^{-1}\right\|_{l(\mathcal{E})} \leq 1$. Therefore,

$$
\begin{aligned}
\left\|x_{n+1}-x_{n}\right\|_{l(\mathcal{E})} \preceq\left(\frac{\frac{M}{2}}{I_{l(\mathcal{E})}-\frac{M}{2}}\right)\left\|x_{n}-x_{n-1}\right\|_{l(\mathcal{E})} \\
\preceq\left(\frac{\frac{M}{2}}{I_{l(\mathcal{E})}-\frac{M}{2}}\right)^{2}\left\|x_{n-1}-x_{n-2}\right\|_{l(\mathcal{E})} \\
\quad \vdots \\
\preceq\left(\frac{\frac{M}{2}}{I_{l(\mathcal{E})}-\frac{M}{2}}\right)^{n}\left\|x_{1}-x_{0}\right\|_{l(\mathcal{E})} .
\end{aligned}
$$

Let $t=\frac{M}{2}\left(I_{l(\mathcal{E})}-\frac{M}{2}\right)^{-1}, B=\left\|x_{1}-x_{0}\right\|_{l(\mathcal{E})}$.

Implies $\left\|x_{n+1}-x_{n}\right\|_{l(\mathcal{E})} \preceq t^{n} B$

For $n+1>m$

$$
\begin{gathered}
\left\|x_{n+1}-x_{m}\right\|_{l(\mathcal{E})} \preceq\left\|x_{n+1}-x_{n}\right\|_{l(\mathcal{E})}+\left\|x_{n}-x_{n-1}\right\|_{l(\mathcal{E})}+\cdots+\left\|x_{m+1}-x_{m}\right\|_{l(\mathcal{E})} \\
\preceq t^{n} B+t^{n-1} B+\cdots+t^{m} B \\
\preceq\left(t^{n}+t^{n-1}+\cdots+t^{m}\right) B \\
=\sum_{k=m}^{n} t^{k} B \\
=\sum_{k=m}^{n} t^{\frac{k}{2}} t^{\frac{k}{2}} B^{\frac{1}{2}} B^{\frac{1}{2}} \\
=\sum_{k=m}^{n} B^{\frac{1}{2}} t^{\frac{k}{2}} t^{\frac{k}{2}} B^{\frac{1}{2}} \\
=\sum_{k=m}^{n}\left(t^{\frac{k}{2}} B^{\frac{1}{2}}\right)^{*}\left(t^{\frac{k}{2}} B^{\frac{1}{2}}\right) \\
=\sum_{k=m}^{n}\left|t^{\frac{k}{2}} B^{\frac{1}{2}}\right|^{2}
\end{gathered}
$$




$$
\begin{aligned}
& \preceq\left\|\sum_{k=m}^{n}\left|t^{\frac{k}{2}} B^{\frac{1}{2}}\right|^{2}\right\|_{l(\mathcal{E})} I_{l(\mathcal{E})} \\
& \preceq \sum_{k=m}^{n}\left\|B^{\frac{1}{2}}\right\|_{l(\mathcal{E})}^{2}\left\|t^{\frac{k}{2}}\right\|_{l(\mathcal{E})}^{2} I_{l(\mathcal{E})} \\
& =\|B\|_{l(\mathcal{E})} \sum_{k=m}^{n}\|t\|_{l(\mathcal{E})}^{k} I_{l(\mathcal{E})} \\
& \preceq\|B\|_{l(\mathcal{E})} \frac{\|t\|_{l(\mathcal{E})}^{m}}{1-\|t\|_{l(\mathcal{E})}^{m}} I_{l(\mathcal{E})} \longrightarrow 0_{l(\mathcal{E})}(m \longrightarrow+\infty),
\end{aligned}
$$

where $I_{l(\mathcal{E})}$ the unite element in $l(\mathcal{E})$, Therefore $\left\{x_{n}\right\}$ is a Cauchy sequence with respect to $l(\mathcal{E})$. By the completeness of $\left(X, l(\mathcal{E}),\|\cdot\|_{l(\mathcal{E})}\right)$, there exists an $x \in X$ such that $\lim _{n \longrightarrow+\infty} x_{n}=\lim _{n \longrightarrow+\infty} T x_{n-1}=x$.

Since

$$
\begin{aligned}
\|T x-x\|_{l(\mathcal{E})} \preceq\left\|T x-T x_{n}\right\|_{l(\mathcal{E})}+\left\|T x_{n}-x\right\|_{l(\mathcal{E})} \\
\preceq \frac{M}{2}\left(\left\|T x-x_{n}\right\|_{l(\mathcal{E})}+\left\|T x_{n}-x\right\|_{l(\mathcal{E})}\right)+\left\|T x_{n}-x\right\|_{l(\mathcal{E})} \\
\preceq \frac{M}{2}\left(\|T x-x\|_{l(\mathcal{E})}+\left\|x-x_{n}\right\|_{l(\mathcal{E})}+\left\|T x_{n}-x\right\|_{l(\mathcal{E})}\right) \\
\quad+\left\|T x_{n}-x\right\|_{l(\mathcal{E})} \\
=\frac{M}{2}\|T x-x\|_{l(\mathcal{E})}+\frac{M}{2}\left\|x-x_{n}\right\|_{l(\mathcal{E})}+\frac{M}{2}\left\|T x_{n}-x\right\|_{l(\mathcal{E})} \\
+\left\|T x_{n}-x\right\|_{l(\mathcal{E})} .
\end{aligned}
$$

Implies $\|T x-x\|_{l(\mathcal{E})} \preceq \frac{\frac{M}{2}}{I_{l(\mathcal{E})}-\frac{M}{2}}\left\|T x_{n}-x\right\|_{l(\mathcal{E})}+\frac{\frac{M}{2}}{I_{l(\mathcal{E})}-\frac{M}{2}}\left\|x-x_{n}\right\|_{l(\mathcal{E})}+\frac{1}{I_{l(\mathcal{E})}-\frac{M}{2}}\left\|T x_{n}-x\right\|_{l(\mathcal{E})}$

$\|T x-x\|_{l(\mathcal{E})} \preceq \frac{\frac{M}{2}}{I_{l(\mathcal{E})}-\frac{M}{2}}\left\|x_{n+1}-x\right\|_{l(\mathcal{E})}+\frac{1}{I_{l(\mathcal{E})}-\frac{M}{2}}\left\|x_{n+1}-x\right\|_{l(\mathcal{E})} \longrightarrow 0(n \longrightarrow+\infty)$

Implies $\|T x-x\|_{l(\mathcal{E})}=0$ implies $T x=x$.

To prove the uniquness suppose that $y(\neq x)$ is another fixed point of $\mathrm{T}$, then

$$
\begin{aligned}
0 & \preceq\|x-y\|_{l(\mathcal{E})}=\|T x-T y\|_{l(\mathcal{E})} \\
& \preceq \frac{M}{2}\left(\|T x-y\|_{l(\mathcal{E})}+\|T y-x\|_{l(\mathcal{E})}\right)
\end{aligned}
$$

Implies $\|x-y\|_{l(\mathcal{E})} \preceq \frac{\frac{M}{2}}{I_{l(\mathcal{E})}-\frac{M}{2}}\|x-y\|_{l(\mathcal{E})}$

Implies \|\|$x-y\left\|_{l(\mathcal{E})}\right\|_{l(\mathcal{E})} \preceq\left\|\frac{\frac{M}{2}}{I_{l(\mathcal{E})}-\frac{M}{2}}\right\|_{l(\mathcal{E})}\|\| x-y\left\|_{l(\mathcal{E})}\right\|_{l(\mathcal{E})} \prec\|\| x-y\left\|_{l(\mathcal{E})}\right\|_{l(\mathcal{E})}$

This means that $\|x-y\|_{l(\mathcal{E})}=0$ implies $x=y$.

Therefore the fixed point is unique. 
Theorem 2. (Extension of Chatterjee Type Theorem) Let $\left(X, l(\mathcal{E}),\|\cdot\|_{l(\mathcal{E})}\right)$ be an $l(\mathcal{E})$ complete normed space and $T: X \longrightarrow X$ be a self mapping satisfy the following contraction condition

$$
\|T x-T y\|_{l(\mathcal{E})} \preceq \frac{M}{3}\left[\quad\|x-y\|_{l(\mathcal{E})}+\|T x-y\|_{l(\mathcal{E})}+\|T y-x\|_{l(\mathcal{E})}\right],
$$

where $M \in(l(\mathcal{E}))_{+}$with $\|M\|_{l(\mathcal{E})}<\frac{3}{4}$, Then T has a unique fixed point.

Proof. Le $x_{0} \in X$ be arbitrary point and construct a sequence $\left\{x_{n}\right\}_{n=0}^{+\infty} \subseteq X$ by the way: $x_{1}=T x_{0}, x_{2}=T x_{1}, \ldots ., x_{n+1}=T x_{n}$.

$$
\begin{aligned}
\| x_{n+1} & -x_{n}\left\|_{l(\mathcal{E})}=\right\| T x_{n}-T x_{n-1} \|_{l(\mathcal{E})} \\
& \preceq \frac{M}{3}\left[\left\|x_{n}-x_{n-1}\right\|_{l(\mathcal{E})}+\left\|T x_{n}-x_{n-1}\right\|_{l(\mathcal{E})}+\left\|T x_{n-1}-x_{n}\right\|_{l(\mathcal{E})}\right] \\
& =\frac{M}{3}\left[\left\|x_{n}-x_{n-1}\right\|_{l(\mathcal{E})}+\left\|x_{n+1}-x_{n-1}\right\|_{l(\mathcal{E})}+\left\|x_{n}-x_{n}\right\|_{l(\mathcal{E})}\right] \\
& \preceq \frac{M}{3}\left[\left\|x_{n}-x_{n-1}\right\|_{l(\mathcal{E})}+\left\|x_{n+1}-x_{n}\right\|_{l(\mathcal{E})}+\left\|x_{n}-x_{n-1}\right\|_{l(\mathcal{E})}\right] \\
& =\frac{M}{3}\left[\quad 2\left\|x_{n}-x_{n-1}\right\|_{l(\mathcal{E})}+\left\|x_{n+1}-x_{n}\right\|_{l(\mathcal{E})}\right] \\
& =\frac{2 M}{3} \quad\left\|x_{n}-x_{n-1}\right\|_{l(\mathcal{E})}+\frac{M}{3}\left\|x_{n+1}-x_{n}\right\|_{l(\mathcal{E})} \quad .
\end{aligned}
$$

Thus,

$\left(I_{l(\mathcal{E})}-\frac{M}{3}\right)\left\|x_{n+1}-x_{n}\right\|_{l(\mathcal{E})} \preceq \frac{2 M}{3}\left\|x_{n}-x_{n-1}\right\|_{l(\mathcal{E})}$.

Since $M \in\left(l(\mathcal{E}) \grave{)}_{+}\right.$with $\left\|\frac{M}{3}\right\|_{l(\mathcal{E})} \leq \frac{1}{4}$, one have $\left(I_{l(\mathcal{E})}-\frac{M}{3}\right)^{-1} \in(l(\mathcal{E}))_{+}$, and furthermore $\frac{M}{3}\left(I-\frac{M}{3}\right)^{-1} \in(l(\mathcal{E}))_{+}$with $\left\|\frac{M}{3}\left(I_{l(\mathcal{E})}-\frac{M}{3}\right)^{-1}\right\|_{l(\mathcal{E})} \leq \frac{1}{2}$, we have that $\| 2\left(\frac{M}{3}\left(I_{l(\mathcal{E})}-\right.\right.$ $\left.\left.\frac{M}{3}\right)^{-1}\right) \|_{l(\mathcal{E})} \leq 1$. Therefore,

$$
\begin{gathered}
\left\|x_{n+1}-x_{n}\right\|_{l(\mathcal{E})} \preceq 2\left(\frac{\frac{M}{3}}{I_{l(\mathcal{E})}-\frac{M}{3}}\right)\left\|x_{n}-x_{n-1}\right\|_{l(\mathcal{E})}=t\left\|x_{n}-x_{n-1}\right\|_{l(\mathcal{E})} \\
\preceq t^{2}\left\|x_{n-1}-x_{n-2}\right\|_{l(\mathcal{E})} \\
\vdots \\
\preceq t^{n}\left\|x_{1}-x_{0}\right\|_{l(\mathcal{E})},
\end{gathered}
$$

where $t=2\left(\frac{M}{3}\left(I_{l(\mathcal{E})}-\frac{M}{3}\right)^{-1}\right)$.

For $n+1>m$. 
R. A. Rashwan et al. / Eur. J. Pure Appl. Math, 14 (4) (2021), 1237-1248

$$
\begin{aligned}
\left\|x_{n+1}-x_{m}\right\|_{l(\mathcal{E})} & \preceq\left\|x_{n+1}-x_{n}\right\|_{l(\mathcal{E})}+\left\|x_{n}-x_{n-1}\right\|_{l(\mathcal{E})}+\cdots+\left\|x_{m+1}-x_{m}\right\|_{l(\mathcal{E})} \\
& \preceq\left(t^{n}+t^{n-1}+\cdots+t^{m}\right)\left\|x_{1}-x_{0}\right\|_{l(\mathcal{E})} .
\end{aligned}
$$

Let $B=\left\|x_{1}-x_{0}\right\|_{l(\mathcal{E})}$

$$
\begin{aligned}
\Rightarrow\left\|x_{n+1}-x_{m}\right\|_{l(\mathcal{E})} & =\sum_{k=m}^{n} t^{k} B \\
& =\sum_{k=m}^{n} t^{\frac{k}{2}} t^{\frac{k}{2}} B^{\frac{1}{2}} B^{\frac{1}{2}} \\
& =\sum_{k=m}^{n} B^{\frac{1}{2}} t^{\frac{k}{2}} t^{\frac{k}{2}} B^{\frac{1}{2}} \\
& =\sum_{k=m}^{n}\left(t^{\frac{k}{2}} B^{\frac{1}{2}}\right)^{*}\left(t^{\frac{k}{2}} B^{\frac{1}{2}}\right) \\
& =\sum_{k=m}^{n}\left|t^{\frac{k}{2}} B^{\frac{1}{2}}\right|^{2} \\
& \preceq\left\|\sum_{k=m}^{n}\left|t^{\frac{k}{2}} B^{\frac{1}{2}}\right|^{2}\right\|_{l(\mathcal{E})} I_{l(\mathcal{E})} \\
\preceq & \sum_{k=m}^{n}\left\|B^{\frac{1}{2}}\right\|_{l(\mathcal{E})}^{2}\left\|t^{\frac{k}{2}}\right\|_{l(\mathcal{E})}^{2} I_{l(\mathcal{E})} \\
= & \|B\|_{l(\mathcal{E})} \sum_{k=m}^{n}\|t\|_{l(\mathcal{E})}^{k} I_{l(\mathcal{E})} \\
\preceq & \|B\|_{l(\mathcal{E})} \frac{\|t\|_{l(\mathcal{E})}^{m}}{1-\|t\|_{l(\mathcal{E})}^{m}} I_{l(\mathcal{E})} \longrightarrow 0_{l(\mathcal{E})}(m \longrightarrow+\infty),
\end{aligned}
$$

where $I_{l(\mathcal{E})}$ the unite element in $l(\mathcal{E})$, Therefore $\left\{x_{n}\right\}$ is a Cauchy sequence with respect to $l(\mathcal{E})$. By the completeness of $\left(X, l(\mathcal{E}),\|\cdot\|_{l(\mathcal{E})}\right)$, there exists an $x \in X$ such that $\lim _{n \longrightarrow+\infty} x_{n}=\lim _{n \longrightarrow+\infty} T x_{n-1}=x$.

Since

$$
\begin{aligned}
\| T x- & x\left\|_{l(\mathcal{E})} \preceq\right\| T x-T x_{n}\left\|_{l(\mathcal{E})}+\right\| T x_{n}-x \|_{l(\mathcal{E})} \\
& \preceq \frac{M}{3}\left(\left\|x-x_{n}\right\|_{l(\mathcal{E})}+\left\|T x-x_{n}\right\|_{l(\mathcal{E})}+\left\|T x_{n}-x\right\|_{l(\mathcal{E})}\right)+\left\|T x_{n}-x\right\|_{l(\mathcal{E})} \\
\preceq & \frac{M}{3}\left(\left\|x-x_{n}\right\|_{l(\mathcal{E})}+\left\|T x-x_{n}\right\|_{l(\mathcal{E})}+\left\|x_{n+1}-x\right\|_{l(\mathcal{E})}\right)+\left\|T x_{n}-x\right\|_{l(\mathcal{E})} .
\end{aligned}
$$

Implies $\|T x-x\|_{l(\mathcal{E})} \preceq \frac{\frac{M}{3}}{I_{l(\mathcal{E})}-\frac{M}{3}}\left(2\left\|x-x_{n}\right\|_{l(\mathcal{E})}+\left\|x_{n+1}-x\right\|_{l(\mathcal{E})}\right)+\frac{1}{I_{l(\mathcal{E})}-\frac{M}{3}}\left\|x_{n+1}-x\right\|_{l(\mathcal{E})} \longrightarrow$ $0($ at $n \longrightarrow+\infty)$.

Then this implies that $T x=x$ i.e., $x$ is fixed point of $T$.

To prove the uniquencess suppose that $y(\neq x)$ is another fixed point of $\mathrm{T}$, then

$$
0 \leq\|x-y\|_{l(\mathcal{E})}=\|T x-T y\|_{l(\mathcal{E})}
$$




$$
\begin{aligned}
& \preceq \frac{M}{3}\left(\|x-y\|_{l(\mathcal{E})}+\|T x-y\|_{l(\mathcal{E})}+\|T y-x\|_{l(\mathcal{E})}\right) \\
& \preceq M\|x-y\|_{l(\mathcal{E})},
\end{aligned}
$$

Implies $0 \leq\|\| x-y\|\|_{l(\mathcal{E})} \leq\|M\| x-y\|\|_{l(\mathcal{E})}<\|\| x-y\|\|_{l(\mathcal{E})}$

This is contradiction implies $x=y$.

Therefore the fixed point is unique.

\section{Conclusions}

In this paper, we introduced the notions of metric space valued-operator of Hilbert $C^{*}$-module. We define some contraction mapping and prove some fixed point theorems (such as Chatterjee and extension of Chatterjee) for a self mappings $\mathrm{T}$ on the Banach space $l(\mathcal{E})$.

\section{References}

[1] Chatterjee, SK. Fixed-point theorems. C. R. Acad. Bulgare Sci. 25, 727-730 (1972).

[2] Douglas, RG. Banach Algebra Techniques in Operator Theory. Springer, Berlin (1998).

[3] Gilbert helmberg. Introduction to spectral theory in Hilbert space. Technological University Eindhoven .

[4] Kadelburg et al, Z . Remarks on the paper "Fixed point theorems for cyclic contractions in $\mathrm{C}^{*}$-algebra-valued b-metric spaces. Adv. Oper. Theory. no. 1, 93-104,1 (2016).

[5] Kaplansky, I. Modules over operator algebras. Amer J. Math., 75, 839-858, (1953).

[6] Kasparov, G.G. Hilbert $C^{*}$-modules. Theorems of Stinespring and Voiculescu, J. Operator Theory 4, 133-150,(1980).

[7] Karapinar, E. Fixed point theorems in cone Banach spaces. Fixed Point Theory Appl. 2009 Article ID 609281, 9 pages, 2009, doi:10.1155/2009/609281

[8] Lance, E. C. Hilbert $C^{*}$-Modules. A Toolkit for Operator Algebraists. Cambridge, England: Cambridge University Press, 1995.

[9] Murphy, G. J. $C^{*}$-algebras and operator theory. Academic Press, 1990.

[10] Mustafa, R. Omran, S. Ngoc, Qn. Fixed point theory using $\psi$ contractive mapping In $C^{*}$-Algebra valued $b$-metric space. Mathematics , 9, 92,(2021). 
[11] $\ddot{O}$ zer, $\ddot{O}$. Omran, S. Common fixed point In $C^{*}$-Algebra b-valued metric space. AIP Conference Proceedings 1773 (1) 050005(2016).

[12] Paschke, W. L. Inner product modules over $B^{*}$-algebras. Trans Amer. Math. Soc.182 , 443-468,(1973).

[13] Priyobarta et al, N. Fixed point theorems on parametric A-metric space, American Journal of Applied Mathematics and Statistics, Vol.6, No. 1, 1-5, 2018.

[14] Qiaoling, X. Lining, J. Zhenhua, Ma. Common fixed point theorems in $C^{*}$-algebravalued metric spaces[J]. Journal of Hubei Normal University(Natural Science), 2015.

[15] Radenović et al,S. Coupled fixed point theorems in $\mathrm{C}^{*}$-algebra-valued b-metric spaces. Scientific publications of the state University of Novi Pazar, Ser. A: Appl. Math. Inform. and Mech. vol. 9,81-90,1 (2017).

[16] Rieffel, M. A. Induced representations of $C^{*}$-algebras. Adv in Math.,13(2), 176$257,(1974)$

[17] Turkoglu, D. Abuloha, M. and Abdeljawad, T. Some theorems and examples of cone Banach spaces. J. Comput. Anal. Appl. 12 4, 739-753, (2010).

[18] Vesna Todorčević, Harmonic Quasiconformmal Mappings and Hyperbolic Type Metrics, Springer Nature Switzerland AG 2019.

[19] Wegge-Olsen, N. E. K-Theory and $C^{*}$-Algebras. A Friendly Approach. Oxford, England: Oxford University Press, 1993.

[20] Zhenhua, Ma. Lining, J. Hongkai, S. $C^{*}$-Algebras-valued metric spaces and related fixed point theorems. Fixed Point Theory Appl. 2014, 206 (2014).

[21] Zhenhua, Ma. Lining, J. $C^{*}$-Algebras-valued b-metric spaces and related fixed point theorems. Fixed Point Theory Appl.1,1-12, (2015).

[22] Zoran Kadelburg, Stojan Radenović, Critical remarks on some recent fixed points results in $\mathrm{C}^{*}$-algebra-valued metric spaces, Fixed Point Theory Appl. (2016) 2016:53. 